Ciência e Natura, Santa Maria v.38 Ed. Especial-

IX Workshop Brasileiro de Micrometeorologia , 2016, p. 21 - 27

Revista do Centro de Ciências Naturais e Exatas - UFSM

ISSN impressa: 0100-8307 ISSN on-line: 2179-460X

\title{
Circulação de brisa e a banda de precipitação na margem leste da baía de Marajó
}

\author{
River breeze circulation and precipitation band on the east bank of the Marajo bay
}

\author{
Abner Pinheiro de Matos $^{1}$ e Julia Clarinda Paiva Cohen ${ }^{2}$ \\ ${ }^{1}$ Universidade Federal do Pará, Belém, Brasil \\ abnermatos@gmail.com \\ ${ }^{2}$ Departamento de Meteorologia, Universidade Federal do Pará, Belém, Brasil \\ jcpcohen@gmail.com
}

\begin{abstract}
Resumo
Na região de Belém, além dos sistemas atmosféricos de grande escala como a ZCIT e de mesoescala como as Linhas de Instabilidade, há também sistemas convectivos forçados localmente que também causam precipitação. Este trabalho estuda um sistema convectivo, observado pela primeira vez nessa região durante a campanha do projeto CHUVA, o qual é denominado por Microlinha de Instabilidade Fluvial(MLF) devido sua formação estar associada a circulação de brisa fluvial. Os dados do satélite GOES 12, radar banda X, anemômetro de hélice, pluviômetros, disdrômetros, reanálises do NCEP foram utilizados para estudar esta MLF. Simulação numérica de alta resolução com o modelo BRAMS foi feita para esta MLF, utilizando-se duas grades com resolução horizontal de $3 \mathrm{Km}$ e $1 \mathrm{Km}$. Os resultados da simulação mostraram que a MLF foi formada através de brisa fluvial proveniente da baía de Marajó, causando precipitação desde sua fase inicial sobre a região de Belém até quando estava sobre a margem oeste da baía do Marajó. Durante sua propagação para Oeste, observou-se aumento da sua intensidade, que foi máxima sobre a baía de Marajó, onde há intenso transporte de cargas e passageiros e naufrágios são o tipo de acidente mais frequente.
\end{abstract}

Palavras-chave: Microlinha. Refletividade. Brisa fluvial. BRAMS.

\begin{abstract}
The precipitation in Belem region is mainly due the large atmospheric systems such as ITCZ and mesoscale systems like the Instability Lines. Beside these systems, there are convective systems forced locally which also promote precipitation in this region. This paper studies a convective system, observed for first time during the campaign of the RAIN project, which is called Micro Instability Line Fluvial (MLF) because its formation is associated with river breeze circulation. The GOES 12 satellite data, radar band X, anemometer, rain gauges, disdrometer, and NCEP reanalyzes were used to study this MLF. High-resolution numerical simulation with the BRAMS model was made for this MLF, using two grids with horizontal resolution of $3 \mathrm{~km}$ and $1 \mathrm{~km}$. The simulation results showed that the MLF was formed by river breeze from the Marajo Bay, causing precipitation since the early stages in Belem area until this MLF was on the west bank of the Marajo bay. During its propagation westward, this MLF had increased its intensity, which was maximum over the Bay of Marajo, where there is intense transportation of cargo and passengers, and shipwrecks are the most frequent type of accidents.
\end{abstract}

Keywords: Micro line. Reflectivity. River breeze. BRAMS. 


\section{Introdução}

Belém, capital do estado do Pará, está localizada aproximadamente a $150 \mathrm{~km}$ da costa atlântica e é banhada pela baia do Marajó.

$\mathrm{O}$ nordeste paraense tem clima quente $\mathrm{e}$ úmido (Peel et al., 2007) e seus níveis de precipitação anual estão entre 2000 e 3000 mm, apresentando duas estações bem definidas: chuvosa, nos meses de Dezembro a Maio e menos chuvosa, de Junho a Novembro (Figueroa e Nobre, 1990). Nesta região a precipitação é originadas através de sistemas convectivos forçados localmente (Silva Dias at al., 2004, Lu et al., 2005, Fitzjarrald et al., 2008, Mesquita e Ramos da Silva, 2012, Cohen et al., 2014), pelas linhas de instabilidade formadas ao longo da costa atlântica (Molion, 1987; Garstang et al., 1994; Cohen et al., 1995, Alcântara et al., 2011) e existem também os sistemas atmosféricos de grande escala tal como a Zona de Convergência Intertropical (ZCIT) que define os períodos chuvoso e menos chuvoso (Ferreira et al., 2015).

Essa região também experimenta a ocorrência de sistemas atmosféricos de mesoescala, como Sistemas Convectivos de Mesoescala Circulares (SCMC) que são facilmente observados por satélite. Em 2011, ano de La Ninã, os SCMC maiores foram mais frequentes e restritos à foz do rio Tocantins e da Baía de Marajó (Sodré, 2013).

As LI são formadas ao longo da costa norte da América do Sul, tem sua formação associada à circulação de brisa marítima, movendo-se para o interior da Amazônia, provocando fortes chuvas, ocorrendo com mais frequência entre Abril e Agosto (Cohen et al., 1995; Alcântara et al., 2011).

Um sistema atmosférico local, como a circulação de brisa fluvial é uma característica notada nos grandes rios da Amazônia, que ocorre devido a diferente capacidade térmica entre a superfície do rio e a superfície do solo, esta mais quente que a do rio durante o dia, com movimento de ar ascendente sobre o solo por causa da baixa pressão atmosférica e fluxo do rio para a terra em baixo nível (Silva Dias et al., 2004).

Na região de Santarém, foi observada a formação de nuvens na margem leste do rio Tapajós, ausentes na margem oeste, atribuída à circulação de brisa fluvial (Sival Dias et al., 2004;
Lu et al., 2005), bem como uma faixa de precipitação ao sul do rio Amazonas, também associada à brisa fluvial (Cohen et al., 2014) .

Em Junho de 2011, com uso de dados de refletividade de radar banda $X$, foi observada pela primeira vez na região metropolitana de Belém, na campanha do projeto CHUVA (Machado et al., 2014), a ocorrência de microlinhas de instabilidade, tanto na presença de linhas de instabilidade associadas a circulação de brisa marítima quanto na sua ausência.

Estas microlinhas apresentavam orientação paralela ou perpendicular em relação à Baía de Marajó, sendo classificadas neste estudo em dois tipos: Microlinhas de Instabilidade Fluviais (MLF) e Microlinhas de Instabilidade Marítimas (MLM). As MLF formavam-se sobre a região metropolitana de Belém e dissipavam-se sobre a Ilha de Marajó, após atravessarem a Baía de Marajó.

As MLF formavam-se mais cedo (entre 15:00 e 17:30 UTC) que as MLM (entre 17:30 e 23:00 UTC), ambas com tempo de vida de aproximadamente 2 horas (enquanto para as linhas de instabilidade clássicas é de 12 horas), comprimento médio de $150 \mathrm{Km}$ e média de velocidade de propagação de $7 \mathrm{~m} / \mathrm{s}$. A média da distância viajada para as MLF foi de $42 \mathrm{Km}$ (Amaral, em preparação) .

Assim, o objetivo deste artigo é estudar um caso de MLF, observada durante o CHUVA, procurando entender sua origem e estrutura, pois sendo um sistema convectivo profundo que atravessa a baia do Marajó, pode ser importante para a região onde a navegação fluvial é considerável.

\section{Dados e Metodologia}

Os dados utilizados foram aqueles coletados durante a campanha do projeto CHUVA em Belém, em Junho de 2011. Entre o conjunto de instrumentos instalados pelo CHUVA, o radar duplamente polarizado banda $\mathrm{X}$ forneceu os dados de refletividade para a visualização das MLF pela primeira vez nesta região.

Os dados de velocidade máxima do vento, coletados por um anemômetro de hélice instalado na torre em Outeiro, permitiram a detecção das circulações quando a MLF formouse na região de Belém, enquanto os pluviômetros e disdrômetros possibilitaram medir sua 
precipitação. Imagens do satélite GOES 12 canal IR, foram úteis para observação da linha de instabilidade.

Com o objetivo de entender a formação da MLF, realizou-se uma simulação de alta resolução com o modelo regional BRAMS, uma adaptação do Regional Atmospheric Modeling System-RAMS(Pielke et al., 1992) às características brasileiras. Equações prognósticas para a temperatura, o vapor d'água, a água líquida (nuvem e chuva), as três componentes da velocidade do vento e cinco espécies de gelo estão incluidas no BRAMS (Cotton et al., 2003).

Foram utilizadas 2 grades, a mais grossa com $148 \times 148 \times 40$ pontos de grade, com espaçamento horizontal de $3 \mathrm{~km}$, enquanto a grade aninhada tem 197x197x40 pontos e espaçamento horizontal de $1 \mathrm{Km}$ (Figura 1). Ambas tem um espaçamento vertical variável, sendo o primeiro de $50 \mathrm{~m}$, com os outros multiplicados sucessivamente pelo fator 1,1 até atingir $2 \mathrm{Km}$ de espaçamento, mantido até o topo do modelo $(\approx 20$ $\mathrm{Km})$.

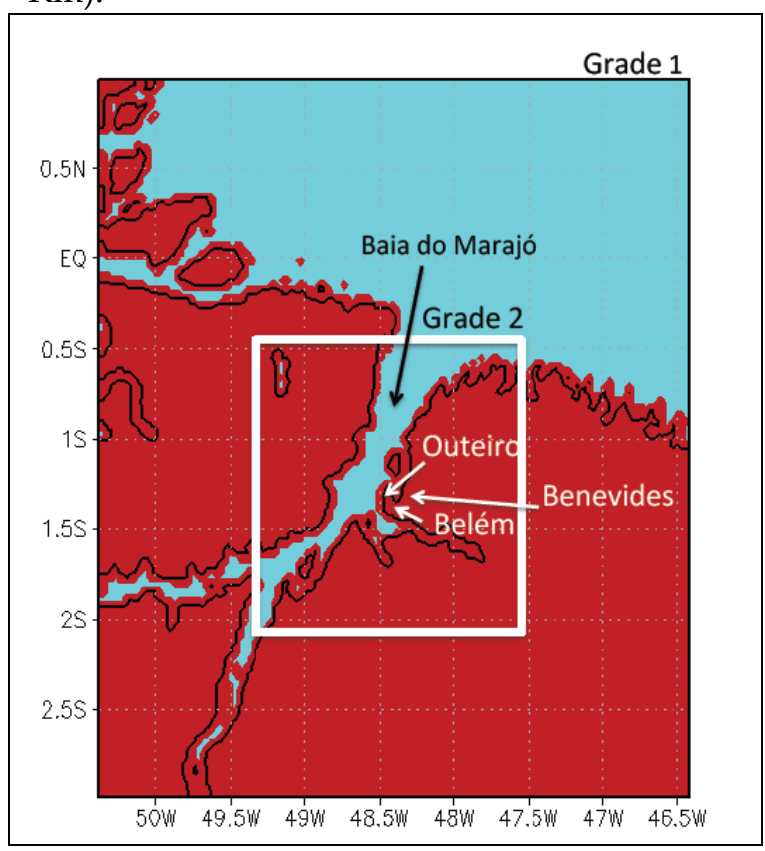

Figura 1: Domínios das grades na simulação da MLF e distribuição dos grande rios e oceano Atlântico.

O tempo de integração do modelo foi de 12 horas com inicio às 12:00 UTC do dia 9 de Junho de 2011. A inicialização do modelo foi heterogênea utilizando as reanálises do NCEP e a temperatura da superfície do mar foi aquela fornecida pelo NOAA correspondente a semana deste estudo. A umidade do solo foi variável e também corresponde aos dias desta simulação. A microfísica de nuvem completa foi acionada em ambas as grades, e não se utilizou a parametrização de cumulus.

\section{Resultados}

\subsection{Observacional}

Durante a campanha do CHUVA em Belém, foram observadas pelo radar um total de 23 microlinhas de instabilidade, sendo 7 fluviais (MLF) e 16 marítimas (MLM). Dos 7 casos de MLF, em 3 não houve linha de instabilidade. Já entre os 16 casos de perpendiculares, em 4 não ocorreram linhas de instabilidade (Amaral, em preparação). Assim, percebe-se que as microlinhas independem da ocorrência de linhas de instabilidade, podendo ocorrer na sua ausência.

A Figura 2 mostra a MLF formada no dia 9 de Junho, quando já estabelecida na região de Belém. A sua formação foi observada às 14:33 (não mostrado), tendo se dissipado às 17:24 sobre a ilha do Marajó (não mostrado). Normalmente estes sistemas de MLF se intensificam sobre a baía do Marajó, durante sua propagação para oeste.

A Figura 3 ilustra um corte transversal da refletividade do radar sobre a MLF na latitude de $1,32^{\circ} \mathrm{S}$ em seus diferentes estágios. Quando este sistema convectivo encontra-se a leste da baía de Marajó (Figuras 3a, 3b), em sua fase inicial o topo da convecção chega até quase $8 \mathrm{Km}$ e quando atravessa a baía de Marajó se intensifica chegando a $13 \mathrm{Km}$ de altura.

A Tabela 1 mostra os totais de precipitação acumulados para o período das 14:00 às 17:00 UTC em que a microlinha passou por cima dos sítios do CHUVA, detectados pelos instrumentos lá instalados

Tanto o pluviômetro 1 quanto o disdrômetro ótico Parsivel mostraram que a MLF passou com mais atividade convectiva sobre Benevides do que em Outeiro (veja localização na Figura 1). Certamente a precipitação deve ter sido maior sobre a baía de Marajó quando a MLF se intensificou, porém não se dispõem de instrumentos sobre a baía de Marajó. 


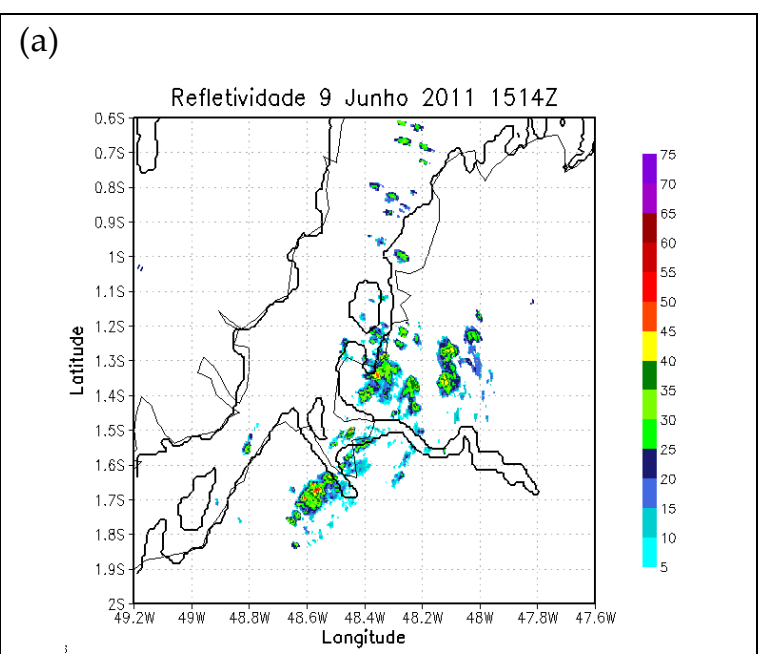

(b)

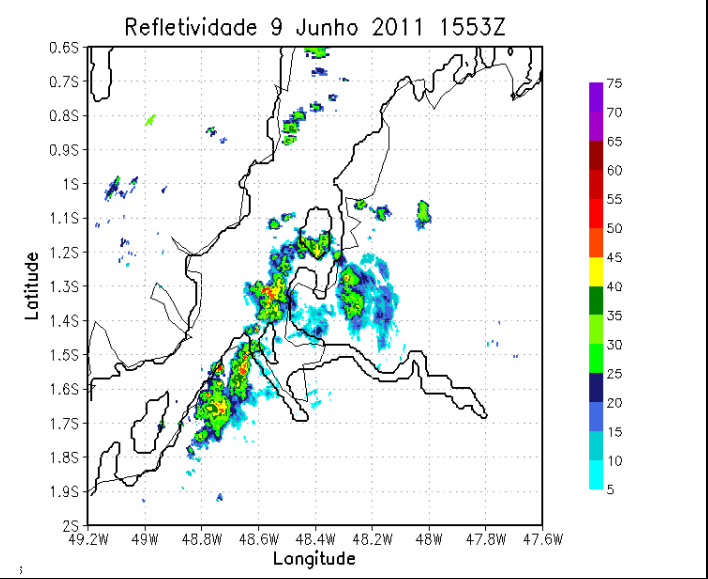

(c)

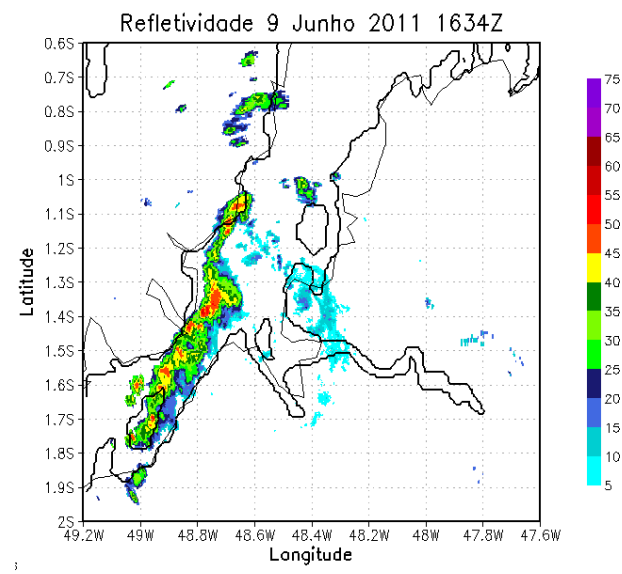

Figura 2: Refletividade do radar na altura de 2 $\mathrm{Km}$, em diferentes estágios da MLF formada no dia 9 de Junho de 2011: (a) às 15:14 UTC; (b) às 15:53 UTC e (c) às 16:34 UTC.

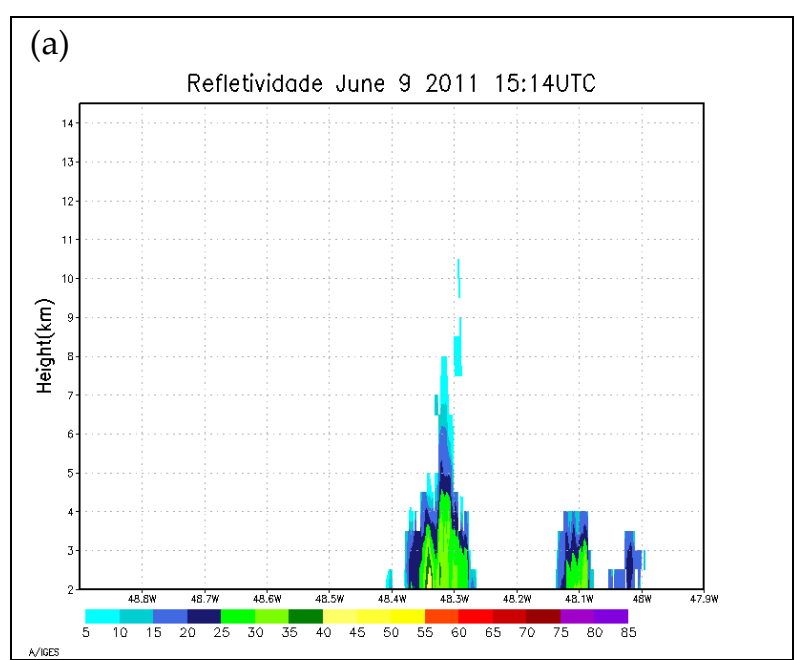

(b)

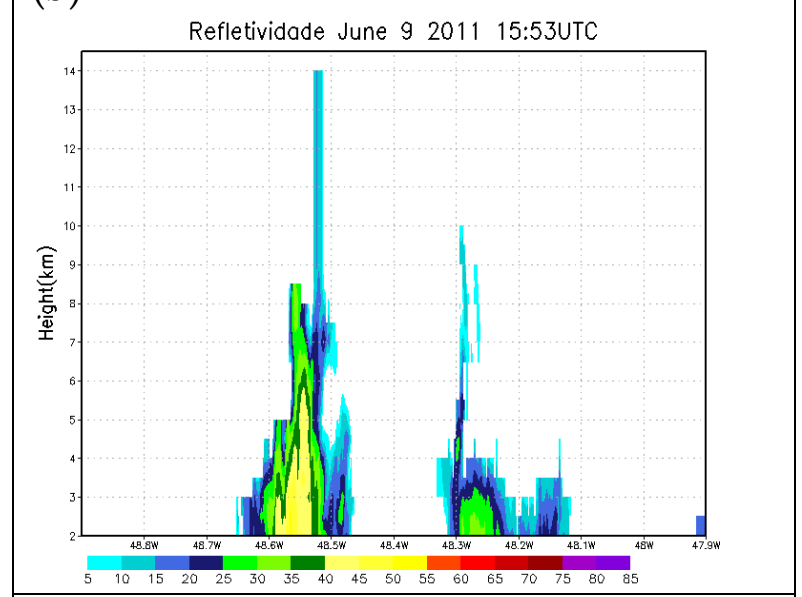

(c)

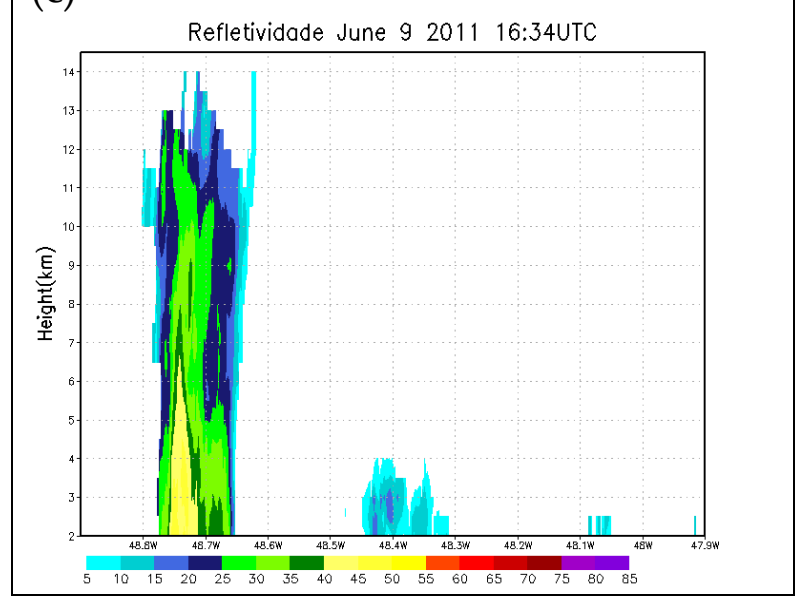

Figura 3: Perfis verticais da refletividade na latitude de 1,32ㅇ em diferentes estágios da MLF formada no dia 9 de Junho de 2011: (a) às 15:14 UTC; (b) às 15:53 UTC e (c) às 16:34 UTC. 
Tabela 1: Precipitação (mm) acumulada entre 14 e 17 UTC do dia 9 de Junho de 2011 durante a passagem da MLF sobre a região metropolitana de Belém

\begin{tabular}{|l|c|c|}
\hline \multirow{2}{*}{ Local } & \multicolumn{2}{|c|}{ Precipitação acumulada (mm) de 14 às 17 UTC } \\
\cline { 2 - 3 } & Pluviômetro 1 & Disdrômetro Parsivel \\
\hline Benevides & 8,382 & 9,37 \\
\hline Outeiro & 1,016 & 2,00 \\
\hline Aeroporto & 0,254 & xxxx \\
\hline
\end{tabular}

A média do vento máximo e das componentes zonal e meridional, para os dias de formação de MLF durante o CHUVA, estão representadas na Figura 4 . O vento máximo aumenta durante o dia e o vento zonal é predominantemente de leste. Enquanto isto, o vento meridional passa a ser de norte entre 17:00 e 21:00 UTC, indicando a entrada de ar vindo da baía de Marajó associado à circulação de brisa fluvial.

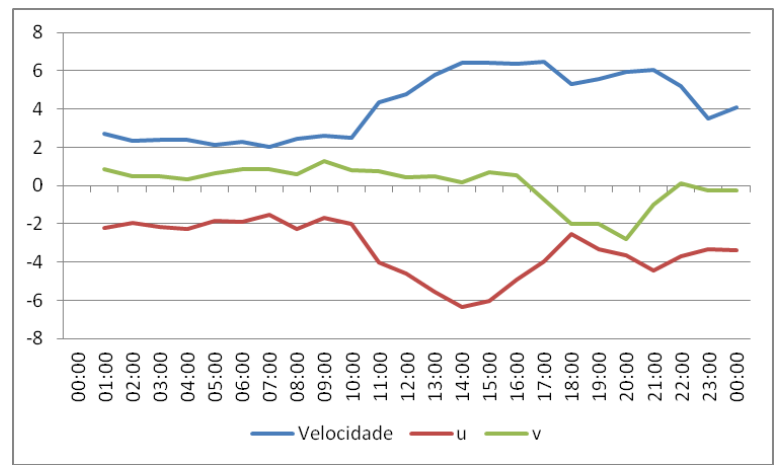

Figura 4: (a) Média horária em $\mathrm{m} / \mathrm{s}$ do vento máximo e suas componentes horizontais em dias com MLF durante a campanha do Chuva.

A distribuição do vento máximo e suas componentes zonal e meridional para o dia da MLF alvo de estudo neste artigo, apresentada na Figura 5, mostra que o vento meridional passou de um vento sul para norte às 14:00 UTC, marcando a entrada da brisa fluvial vindo da baía de Marajó. É possivel que esta brisa possa confluir com o vento de leste sobre o continente dando assim origem a formação da MLF sobre o continente na margem leste da baía de Marajó.

$\mathrm{Na}$ região de Santarém, as séries de dados obtidos através de estações meteorológicas automáticas instaladas próximo ao rio e outras mais no interior do continente, mostraram que em situações de ventos alísios fracos, o vento associado à circulação de brisa fluvial tem seu sentido invertido, ou seja, durante o dia o vento está no sentido do rio para o continente, enquanto no período noturno ele está no sentido oposto (Fitzjarrald et al., 2008).

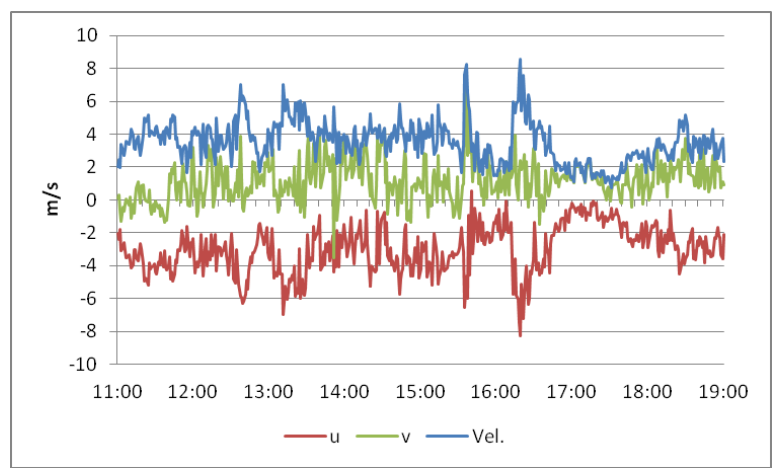

Figura 5: Distribuição do vento máximo e componentes zonal e meridional em Outeiro, a cada minuto, entre 11:00 e 19:00 UTC do dia 9 de Junho de 2011.

\subsection{Simulação}

A Figura 6 apresenta a taxa de precipitação e o vento no nível de 24,4 metros às 20:00 UTC do dia 9 de Junho. É possível observar a canalização do vento na baía de Marajó, sendo que este vento penetra no continente através da margem leste da baía e converge com o escoamento de Leste sobre o continente. Assim, desta confluência da brisa fluvial com o escoamento de Leste há o levantamento de ar com a consequente formação da MLF. Este aspecto pode ser observado na Figura 7, onde se observa a circulação de brisa entrando sobre o continente na margem leste da baía. Verifica-se também que a profundidade desta convecção simulada é semelhante ao observado no radar quando a MLF está sobre o continente (Figuras 3a, 3b). Para a região de Santarém a profundidade da nuvem convectiva foi menor (Silva Dias et al., 2004).

Ao comparar os resultados desta simulação com os resultados observados no radar, percebese que o modelo conseguiu capturar a formação e estrutura da MLF, embora com atraso de cerca de 5 horas.

Entre 2008 e 2013 a baía de Marajó apresentou 17 acidentes, sendo 12 naufrágios, com 7 destes causados por ventos fortes e ondas(Santos, 2015). É possível que a MLF aumente o risco desses naufrágios, uma vez que os ventos aprximaramse de 10 m/s na simulação (ver Figura 6). 


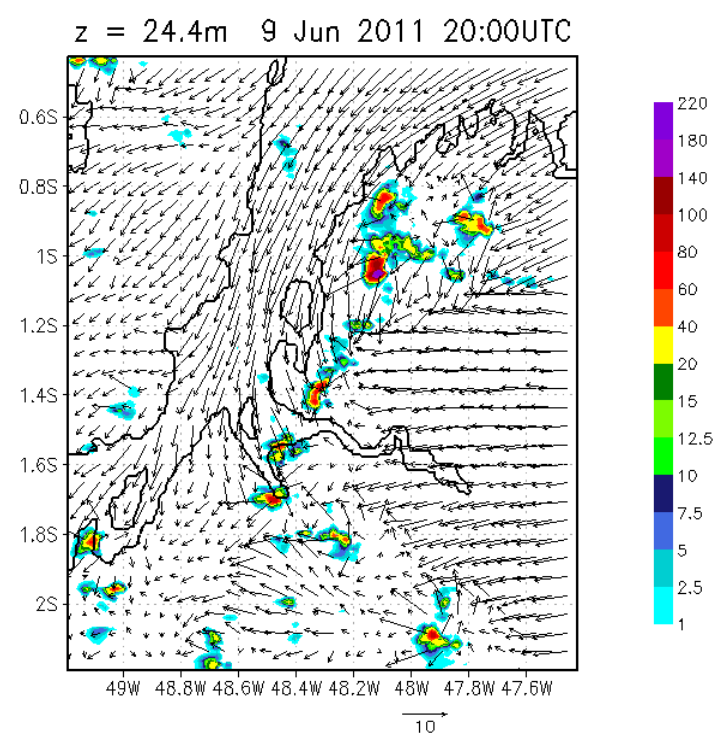

Figura 6: Vento horizontal $(\mathrm{m} / \mathrm{s})$ no nível de 24,4 metros e taxa de precipitação $(\mathrm{mm} / \mathrm{h})$ na grade $2(1$ $\mathrm{km})$.

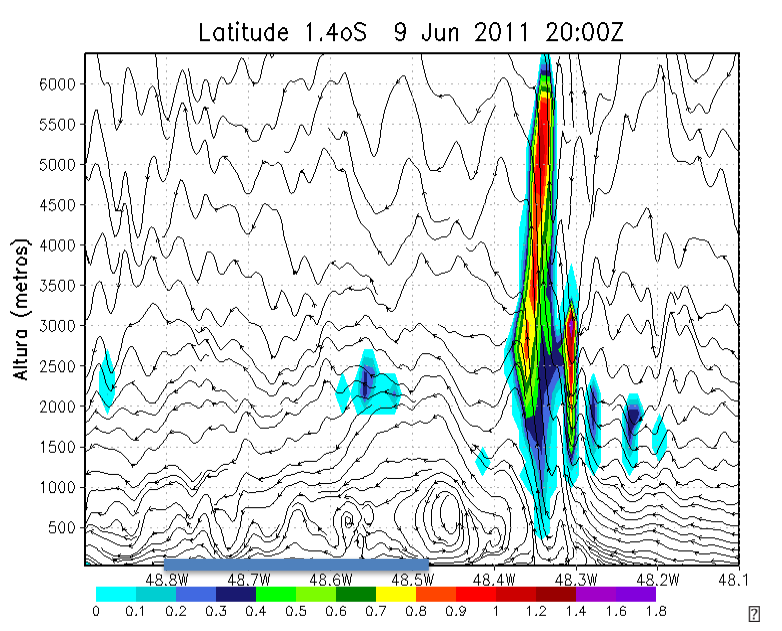

Figura 7: Perfil vertical em 1,4을 às 20:00 UTC do dia 9 de Junho de 2011 para as linhas de corrente de $\mathrm{u}, \mathrm{w}$ e razão de mistura do condensado da nuvem (sombreado). A barra azul, entre $48,8 \mathrm{~W}$ e aproximadamente $48,48 \mathrm{~W}$ representa a baía de Marajó.

\section{Conclusões}

A instalação do radar banda $X$ durante a campanha do Projeto CHUVA, na região de Belém, permitiu observar pela primeira vez a formação de uma banda de convecção paralela à baía de Marajó. Esta banda de convecção foi denominada Micro Linha Fluvial (MLF). Através dos dados de anemômetro observou-se que nos dias de formação destes sistemas convectivos há o estabelecimento de brisa fluvial vindo da baía de Marajó. Esta brisa fluvial encontra o escoamento de leste sobre o continente, onde há convergência, movimento ascendente do ar com consequente formação de convecção em um formato de uma micro linha sobre o continente e paralela à baía de Marajó.

Esta MLF em sua fase inicial atinge a profundidade de $6 \mathrm{Km}$ e ao propagar para oeste se intensifica sobre a baia do Marajó atingindo 13 $\mathrm{Km}$ de profundidade.

A simulação de alta resolução com o modelo BRAMS, mostrou a brisa fluvial confluindo com o escoamento de leste culminando com a formação da MLF, embora com um atraso de aproximadamente 5 horas.

Dado que este sistema se intensifica sobre a baía de Marajó onde há constante navegação fluvial, considera-se de grande importância o seu monitoramento com vista à segurança para a população que transita nesta região.

\section{Agradecimentos}

O autores agradecem à Professora Isabel Vitorino pelas sugestões recebidas Abner Pinheiro de Matos agradece à Professora Júlia Cohen pela disponibilidade de atenção e oportunidade de trabalhar com este objeto de estudo. Tmbém agradece ao Programa de Pós Graduação em Ciências Ambientais da UFPA pela formação e ao CNPq pela bolsa de Mestrado. Os autores agradecem ao apoio da FAPESP na execução do Projeto Chuva (Processo No. 2009/15235-8).

\section{Referências}

ALCÂNTARA CR, SILVA DIAS MAF, SOUZA EP, COHEN JCP. Verification of the role of low level jets in Amazon squall lines. Atmospheric Research. 2011;100:36-44.

COHEN JCP, SILVA DIAS MAF, Nobre CA. Environmental conditions associated with amazonian squall lines: a case study. Mon. Wea. Rev. 1995;123:3163-3174.

COHEN JCP, FITZJARRALD DR, D'OLIVEIRA FAF, SARAIVA I, BARBOSA IRS, GANDU AW, et al. Radar-observed spatial and temporal rainfall variability near the Tapajós-Amazon confluence. RBMET. 2014;29(n. esp.):23-30. 
COTTON WR, PIELKE RA, WALKO RL, LISTON GE, TREMBACK CJ, JIANG H. RAMS 2001: Current status and future directions. Meteorol. Atmos. Phys., Austria. 2003;82(1-4):529

FIGUEROA SN E NOBRE CA. Precipitations distribution over central and western tropical South America. Climanálise - Boletim de Monitoramento e Análise Climática. 1990;5(6):3645.

FITZJARRALD DR, SAKAI RK, MORAES OLL, DE OLIVEIRA RC, ACEVEDO OC, MATTHEW J et al. Spatial and temporal rainfall variability near the Amazon-Tapajós confluence. JGR: Biogeosciences. 2008;113(G1):1-17.

GARSTANG M, MASSIE HL, HALVERSON J, GRECO S. Amazon coastal squall lines. Part I: Structure and Kinematics. Mon. Wea. Rev. 1994;122:608-622.

LU L, DENNING AS, SILVA DIAS MAF, SILVA DIAS P, LONGO M, FREITAS SR, SAATCHI S. Mesoscale circulations and atmospheric $\mathrm{CO}_{2}$ variations in the Tapajós region, Pará, Brazil. JGR. 2005;110(D21102):1-17.

MACHADO LAT, SILVA DIAS MAF, MORALES C, FISCH G, VILA D, ALBRECHT R et al. The chuva project: How does convection vary across Brazil? Bull. Amer. Meteor. Soc. 2014;1365-1380.

MESQUITA RLC E SILVA RR. Insights of meso and micro-scale processes for the Caxiuanã forest region from high resolution simulation. RBMET. 2012;27(2): 253-261.

MOLION LCB. Climatologia dinâmica da região amazônica: Mecanismos de prcipitação. RBMET. 1987;2:107-117.

PEEL MC, FINLAYSON BL, MCMAHON TA. Updated world map of the Köppen-Geiger climate classification. HESS. 2007;11:1633-1644.

PIELKE RA, COTTON WR, WALKO RL, TREMBACK CJ, LYONS WA, GRASSO LD et al. A comprehensive meteorological modeling system - RAMS. Meteorol. Atmos. Phys. 1992;49:69-91.

SANTOS SHM. Acidentes com transportes hidroviários e os extremos meteorológicos no nordeste da Amazônia [dissertação]. Belém: Universidade Federal do Pará; 2015. 92 p.
SODRÉ GRC. Estudo da convecção de mesoescala em diferentes superfícies na amazônia oriental [dissertação]. Belém: Universidade Federal do Pará; 2013. 65 p.

SILVA DIAS MAF, SILVA DIAS PL, LONGO M, FITZJARRALD DR, Denning AS. River breeze circulation in eastern Amazonia: observations and modeling results. Theor. Appl. Climatol. 2004;78(1):111-121. 